\title{
Area-luminance effects and the visual evoked brain response
}

\author{
GARY KRESS \\ Regis College, Denver, Colorado 80221
}

\begin{abstract}
Psychophysical and electrophysiological studies report that luminance must be systematically increased as stimulus area is decreased if a constant response is to be elicited. The visual evoked brain response (VEBR) was recorded from three human subjects as a function of eight stimulus intensities for five different areas. The results indicate: (a) the amplitude of the B-C component of the VEBR increases in a linear fashion as a function of increases in log luminance, (b) there is a linear reciprocal relationship between the magnitude of $\log$ area and $\log$ luminance for targets up to $10 \mathrm{deg}$, and (c) substituting total number of receptors in an area for stimulus size results in receptor-luminance functions identical to the corresponding area-luminance function. Results are discussed in terms of neural summation.
\end{abstract}

One of the basic problems in vision research concerns the relationship between the size of a stimulus and the luminance necessary to produce a given response. Historically, this problem has been investigated using either psychophysical methods (Graham \& Bartlett, 1939; Graham, Brown \& Mote, 1939; Hillman, 1958) or electrophysiological response measures such as the electroretinogram (ERG) (Armington, Tepas, Kropfl, \& Hengst, 1961; Armington \& Thiede, 1954; Boynton \& Riggs, 1951). The psychophysical studies have been concerned mainly with visual absolute threshold determinations in which area and luminance are varied in order to produce a just visible stimulus. The electrophysiological measures have required the use of high-intensity stimuli in order to establish what combination of area and intensity are necessary to produce an electrical response of a certain size.

At the psychophysical absolute threshold level, the relationship between area and luminance has been quantified in a reciprocal relationship expressed as Ricco's law for foveal stimulation and Piper's law for peripheral retinal stimulation. Early studies by Graham et al. (1939) showed that these laws were valid for only a limited range of areas for both the periphery and fovea. Their results showed that as the area of the stimulus increases, the luminance necessary to achieve threshold decreases. A plot of their data using log intensity and $\log$ radius coordinates showed that the area-intensity curves were not linear but decreased in slope as they went from smaller areas to larger areas. A simple power function could not adequately describe the area-luminance relation.

Later electrophysiological studies by Boynton and Riggs (1951) used the ERG to investigate the

This research was supported in part by NIMH Grant MH 14020-03 to Donald 1. Tepas, Saint Louis University, St. Louis, Missouri 63103. area-luminance relationship. The areas studied ranged from 1.25 to $12 \mathrm{deg}$ and were centered on the fovea. Their results showed that there was a linear reciprocal relationship between the magnitude of $\log$ area and $\log$ luminance. The negative slopes of the area-luminance functions varied between .95 and .99 for the three subjects. A surprising finding of this study was that the same response could be obtained if the stimulus was centered on the blind spot. Thus the authors concluded that the results were probably due to light scatter rather than to the calculated focal illumination.

Few studies have examined the area-luminance relationship using the human visual evoked brain response (VEBR). Tepas and Armington (1962) compared the ERG and VEBR methods and found a linear area-luminance relationship using the ERG, while the VEBR data showed that the relationship tended to be curvilinear. Armington (1968) found that the amplitude of both the ERG and VEBR waveforms increased in a linear fashion as a function of increases in area when luminance was held constant. One problem with both of these studies is the fact that only a small number of stimulus presentations was recorded. At moderate light intensities, the ERG is well defined using only about 50 stimulus presentations, while to obtain a representative VEBR, several hundred presentations are probably necessary. Tepas (1974) presents a good discussion on the relationship between EBR variability and sample size.

The purpose of the present study was to investigate the area-luminance relationship using the human VEBR as the investigative tool. More parametric data is needed to validate the VEBR technique and to make comparisons with the area-luminance relationships found using other methods.

\section{METHOD}

\section{Subjects}

Three subjects were used, two male and one female, ranging in age from 18 to 27 years. All subjects had at least $20 / 20$ vision and 


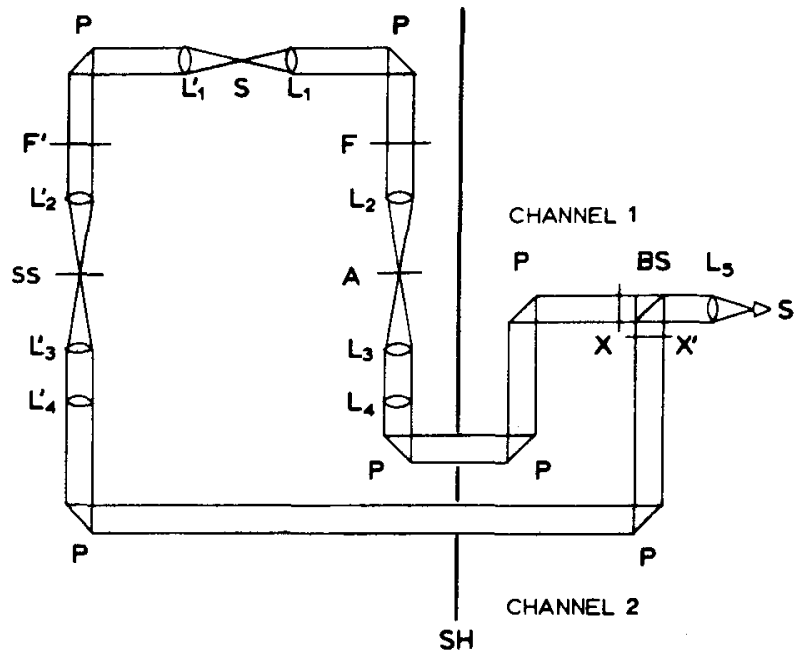

TWO-CHANNEL MAXWELLIAN-VIEW SYSTEM

Figure 1. Two-channel Maxwellian view optical system. See text for explanation.

normal color perception. The subjects were selected on their ability to adapt to the prolonged experimental situation and to show VEBRs which were relatively artifact free and could be reliably measured.

\section{Stimulation Apparatus}

Figure 1 shows schematically the two-channel Maxwellian view optical system which was used to present the stimulus to the subject. The main components of the system were mounted outside an electrically shielded chamber (SH) (Ace Model R $10 \mathrm{H68}$ ). Channel 2 delivered the stimulus target to the subject's right eye and Channel 1 provided the surround illumination. For a detailed description of the optical system, see Tepas (1974).

The stimulus, delivered via the optical system, consisted of a square-wave flash of white light with a duration of $30 \mathrm{msec}$. The rise-fall time of the flash was $6 \mathrm{msec}$. The rate of stimulation was once per second. Five stimulus targets were used, subtending visual angles of $0.75,1.5,3.0,6.0$, and $10 \mathrm{deg}$. With no neutral density filters in Channel 2 and Channel 1 blocked off, the maximum available luminance was $34,890 \mathrm{~mL}$. The surround subtended a visual angle of $16 \mathrm{deg}$. A subjective luminance match, between the target and the surround, could be obtained by placing a $3.5 \log$ unit neutral density filter in the target channel. The luminance of the surround was maintained by placing a monopass interference filter at $F$ in Channel 1 . The peak transmission of the filter was at $400 \mathrm{~nm}$ with a half bandwidth of $21 \mathrm{~nm}$. The purpose of the surround illumination was to reduce the effects of stray light on the VEBR. The intensity of the stimulus was varied in $0.5 \log$ unit steps with neutral density filters placed in Channel 2.

\section{Recording Apparatus}

The EEG was recorded from $O_{z}$ (Jasper, 1958) using Grass E5S chloridated silver-silver electrodes. The $\mathrm{O}_{\mathrm{z}}$ electrode was referred to a linked ear electrode (Beckman biopotential) and the subject was grounded at the forehead. Resistance of the electrodes was kept under $10 \mathrm{k} \Omega$.

The EEG was recorded using a Grass Model 7 polygraph equipped with a wideband ac EEG preamplifier (Model 7P5A) and dc driver amplifiers (Model 7DA). The sensitivity of the ac preamplifier was set at $30 \mu \mathrm{V} / \mathrm{cm}$. The bandpass of the amplifier output to the computer was 0.15 to $500 \mathrm{~Hz}$.

The amplified potentials from the polygraph driver amplifier was fed into the AX08 analog inputs of the DEC Lab-8 computer system. The VEBRs were computed using the DEC advanced averager program (DEC-LB-U18C-PB). The Lab-8 system computed the VEBR according to the following specifications: Computations were made at 500 time points distributed over a 500 -msec analysis time. The computer sweep began $50 \mathrm{msec}$ before the onset of the stimulus. Each VEBR consisted of the sum of 120 individual responses.

In addition to the VEBRs recorded in each session, a microvolt calibration signal was also recorded either before or after a session in the same way as the VEBRs. The calibration signal was a 100-msec square-wave output of a Grass SD5 stimulator reduced to the $10 \mu \mathrm{V}$ level. The averaged calibration signal was used to determine the amplitude of the VEBRs.

\section{Procedure}

Each subject was tested for 90 min during 15 separate sessions, each usually 3 to 4 days apart. During each session, the subject was presented with eight luminances at only one area. Each of the luminances was randomly presented twice per session. The area of the target was randomly varied across days. There were three sessions per stimulus area.

The stimulus was delivered to the right eye of the subject while the left eye was covered with an eyepatch. The experimental session began after 8 min of dark adaptation. All subjects were instructed to minimize body movements during a trial and to fixate on the center of the stimulus target. With targets over $3.0 \mathrm{deg}$, a tiny fixation point was provided in the center of the stimulus. Each trial took $120 \mathrm{sec}$. After the trial, the digital values of the summed VEBR were punched out on paper tape using a high-speed punch.

Each combination of area and luminance tested resulted in a total of six VEBR waveforms. In order to increase the signal-to-noise ratio, the six data tapes within each area-luminance combination were added together by means of a program, written in our laboratory, which sums each discrete data point on the VEBR waveform. Thus, for each combination of area and luminance, there was one representative VEBR that was the sum of 720 stimulus presentations.

\section{RESULTS}

Figure 2 shows an example of the VEBRs obtained from one subject for the $0.75-\mathrm{deg}$ area and eight luminances. Positive polarity at the $\mathrm{O}_{z}$ electrode results in an upward deflection. In these waveforms, a small negative deflection (B) occurs between

$$
\text { SUBJECT G-K } \quad .75^{\circ} \quad \text { OZ-EARS }
$$

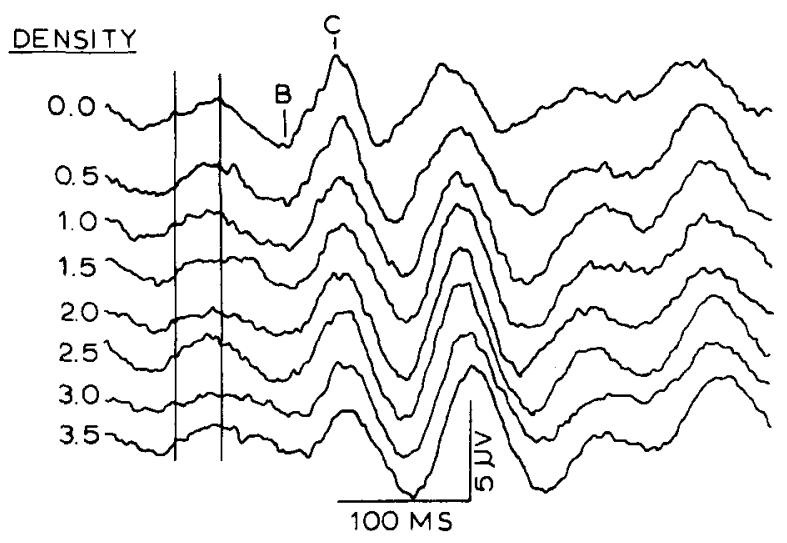

Figure 2. Sample VEBRs for one subject and one stimulus area. Double lines indicate onset and termination of stimulus flash. Positive deflection up. 0.0 density is maximum luminance. 


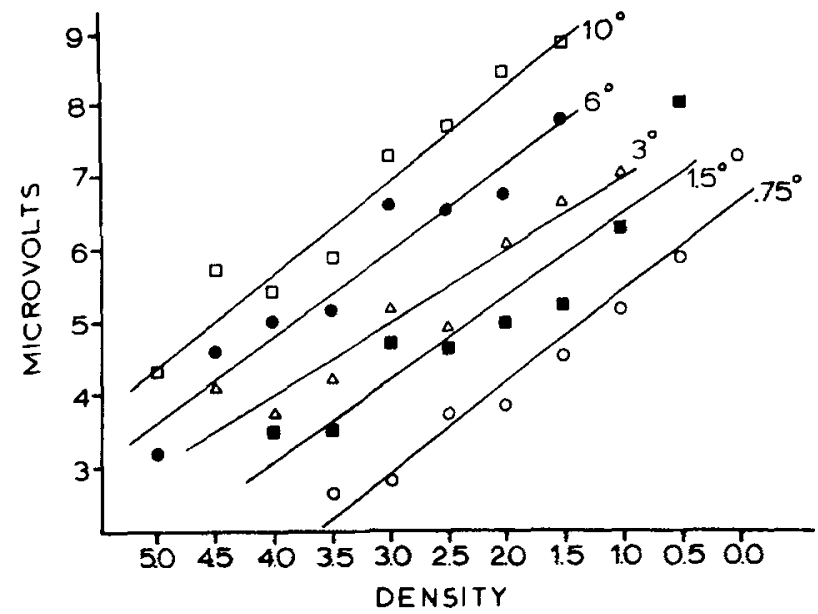

Figure 3. Combined B-C amplitude by luminance functions. Least squares linear regression lines have been fit for each area.

90-100 msec and a large positive deflection (C) occurs at around $120 \mathrm{msec}$. The $\mathrm{B}$ and $\mathrm{C}$ components could easily be identified in all of the waveforms.

Changes in the VEBR as a function of area and luminance were assessed by measurements of the peak-to-peak amplitude of the B-C component. Accurate amplitude measures were obtained by printing out the digital values of the 500 points contained in each waveform. After the values of B and $C$ were identified, they were subtracted from each other and this value was converted to microvolts by comparing it with the calibration signal.

In the preliminary analysis of the data, the B-C amplitude in microvolts for each subject was plotted as a function of log luminance for each of the stimulus areas. Linear regression lines were fitted to the data by least squares computation, and these functions were all statistically significant for linear fit $(p<.01)$. All the functions showed a systematic increase in amplitude as a function of both area and luminance. With the exception of one intensity function (3-deg area) for one subject, tests showed that there were no significant differences $(p>.05)$ between the slopes of any of the functions within subjects. It was therefore considered appropriate to combine the data across subjects to get representative amplitude by luminance functions from which area-luminance functions could be derived.

Figure 3 depicts the combined amplitude by luminance data which are plotted in linear-log coordinates. The computed linear regression functions have been drawn in for each area, and they are statistically significant for linear fit $(p<.001)$. There are no statistically significant differences between the slopes of any of the functions $(p>.05)$. The combined functions clearly show that there is a systematic increase in response amplitude as a function of an increase in luminance for all areas. In addition, the effects of stimulus size are also evidenced by the fact that the intensity functions, for increasingly larger areas, are systematically shifted up on the intensity axis.

In order to look specifically at the relationship between area and luminance, area-luminance functions were derived from Figure 3. These functions were obtained by selecting criterion response levels (in microvolts) on the ordinate of Figure 3 and then determining what combinations of area and luminance were necessary to elicit this criterion response. Figure 4 shows the combinations of area and luminance necessary to elicit criterion responses at five different criterion response levels ranging from 4.5 to $6.5 \mu \mathrm{V}$. Again, linear regression lines have been fit to the data and they show a significant linear fit $(p<.001)$. The slopes of the functions are almost identical, ranging between -.70 and -.72 . Since the functions are plotted in $\log \cdot \log$ coordinates, they could be described by a power function with the exponent corresponding to the slope of the line. Figure 4 shows that as the size of the stimulus is decreased, systematically greater amounts of light are necessary to elicit a criterion response. The values of the slopes indicate that area and luminance are not equally effective, but rather that an increase in area has a greater effect than an increase in luminance in eliciting the response. It is also important to note that the slopes of the derived area-luminance functions do not appreciably change as a function of different criterion response levels. This indicates that the amplitude by luminance functions, shown in Figure 3, are quite similar across the different areas.

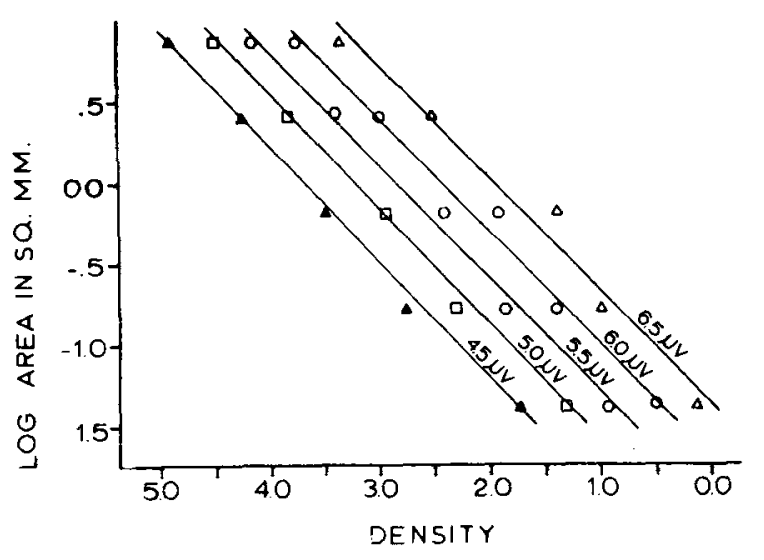

Figure 4. Area-luminance curves for different criterion response levels. Least squares linear regression lines have been fit for each criterion level. Log area was calculated using the assumption that a visual angle of $1 \mathrm{~min}$ corresponds to a distance of $0.005 \mathrm{~mm}$ on the retina (DeVoe et al., 1968). 


\section{DISCUSSION}

The data show that the amplitude of the B-C component of the visual evoked brain response, recorded at $\mathrm{O}_{\mathrm{Z}}$, is a sensitive measure which responds to changes in both area and luminance of stimulation. Within the range of luminances tested, as the luminance of the stimulus increases logarithmically, the amplitude of the B-C component also increases, and this relationship can be described by a linear function. With the intensity levels used in the present study, the data show no evidence that the amplitude of the response levels off or declines at higher luminance levels. Previous studies (Armington, 1968; Vaughan, 1966) show a saturation effect at higher intensities; however, their maximum luminance levels were considerably greater than those used here.

A point needs to be emphasized with regard to the VEBR amplitude-luminance functions. The linear functions obtained are plotted in log-linear coordinates, thus suggesting a Fechner type of logarithmic relationship between stimulus magnitude and amplitude of the VEBR. Though this is one interpretation of the data, it should be noted that $\log$-log fits of the same data produce nearly identical results in terms of linear fit. Therefore, the data could also be used to support a power law interpretation. Previous experiments in our laboratory (Tepas, Boxerman, \& Anch, 1972; Tepas, Guiteras, \& Klingaman, 1974) that investigated both visual and auditory EBR intensity functions have reported similar findings. That is, the intensity functions reported were linear in both log-linear and $\log$-log coordinates. The exact mathematical function which describes the relationship between VEBR amplitude and stimulus intensity, across a broad range of luminances, is an empirical matter which needs further investigation. Because of the limited range of intensities used in the present study, it would not be appropriate to suggest that one function is a better description of the data than another. The coordinate systems used in the present study were chosen mainly because they are consistent with the manipulations of similar data reported in previous studies. The significance of the present data is that it shows that the VEBR technique is a sensitive indicator of brain activity which changes lawfully as a function of visual sensory stimulation.

\section{Area-Luminance Relationships}

Using the criterion response method to compare area with luminance, the data indicate that, within the range of stimuli investigated, there is a linear inverse relationship between area and luminance which can be described by a simple power function.

Previous psychophysical studies (Graham et al., 1939; Graham \& Bartlett, 1939) reported curvilinear relationships between area and luminance for foveal stimulation which could not be described by a simple power function and linear relationships for peripheral stimulation. Hillman (1958) also found a curvilinear relationship between area and luminance for foveal stimulation; however, she pointed out that the degree of curvature varied among her subjects and was more nearly linear than that of Graham, Brown, and Mote. Using higher luminance levels and pupillary constriction as a response measure, Page (1941) reported linear area-luminance functions both in the fovea and the peripheral retina. The present study suggests that for stimuli ranging up to $10 \mathrm{deg}$, stimulating both the fovea and parafovea simultaneously, the relationship between area and luminance is linear. It might also be suggested that a curvilinear relationship may be characteristic only of luminance values at the absolute threshold level.

The present data compare well to the area-luminance functions obtained using the electroretinogram (Boynton \& Riggs, 1951; Tepas \& Armington, 1961) in terms of the linear relationship between area and luminance. The functions presented here are identical in shape to those reported by Boynton and Riggs (1951) for the same range of areas; however, their reported slopes were unity for the stimuli centered on the fovea.

In summary, the combined data from the relevant studies suggest that in order to elicit either a psychophysical absolute threshold response or a neural-electrical response of some criterion value, the area and luminance parameters of the stimulus are related in such a manner that a decrease in luminance can be compensated for by an increase in the area of stimulation. The exact quantitative specification of this relationship awaits further data collection; however, it would seem at the present time that increases in area have a somewhat greater effect than increases in luminance. The latter statement is supported by psychophysical data (Graham et al., 1939), ERG data (Armington et al., 1961), and the present study.

It is tempting to speculate on the underlying neuronal activity that is being measured in all of these studies. A parsimonious explanation of arealuminance effects might be based on a spatial summation or neural integration hypothesis. This hypothesis would simply suggest that the neural response to photic stimulation is the net result of the integration and algebraic summation of the activity of all the individual receptors in the focal area of stimulation. In this proposal, no distinction would be made between rod and cone activity. Luminance levels in the mesopic and photopic range, such as those used in the present study, probably do not produce differential rod and cone activity as suggested by the duplicity theory. A neural summation theory would suggest that an increase in the area of stimulation would require less light to elicit a criterion response 
because more receptors would be available to contribute to the response. Walters (1971) suggests that both photopic and scotopic mechanisms operate in the mesopic range of luminance. Furthermore, he proposes that photopic light pulses, with durations between 25 and $45 \mathrm{msec}$, produce both rod and cone activity which reaches the ganglion cell. The photopic latency can be up to $100 \mathrm{msec}$ faster than the scotopic latency, and thus the inhibitory influence of the cone activity could wane sufficiently to let both rod and cone activity produce impulses in the ganglion cell. The interaction between rod and cone activity that occurs would serve to enhance the ganglion cell activity. It is presumably this ganglion cell activity which is being measured in the VEBR.

It is important to keep in mind that a factor which can influence spatial summation effects is light scatter to nonfocal areas of stimulation. The ERG studies have provided strong evidence for stray light effects by demonstrating that an ERG response can be recorded for light stimulation focused on the blind spot. The present study attempted to control for stray light effects by using the luminous surround.

Although there is no direct way in the present study to test for the contributions of rod activity per se, an indirect way to determine if there is an additive relationship between the number of receptors in the focal area of stimulation and luminance would be to plot the luminance necessary to elicit a criterion response against the number of receptors available in different size areas. A linear reciprocal relationship between total receptors and luminance would result if there was not a differential effect for rod and cone activity. This was done using the data from the present study. An estimate of the number of receptors in each area was made using the receptor counts provided by Østerberg (1935).

Figure 5 shows two graphs obtained by plotting the $\log$ value of the number of receptors in each of the five stimulus areas against the log density necessary to achieve a criterion response of $5.5 \mu \mathrm{V}$. Linear regression equations have been fit to the points. In the lower graph, just the number of cones in the focal area of stimulation are plotted as a function of luminance. If there were a direct reciprocal relationship between the number of cones in an area and the luminance required to elicit a criterion response, then the function describing the points in the graph would be a straight line with a slope of -1 . As the figure shows, the function that best describes the points is linear; however, it has a slope of -.44, indicating less than perfect summation.

The upper graph in the figure shows the results of plotting the log value of the total number of both rods and cones in an area against log luminance. Again, a linear function can be used to describe the points. The slope of this function, however, is -.73 , indicating a

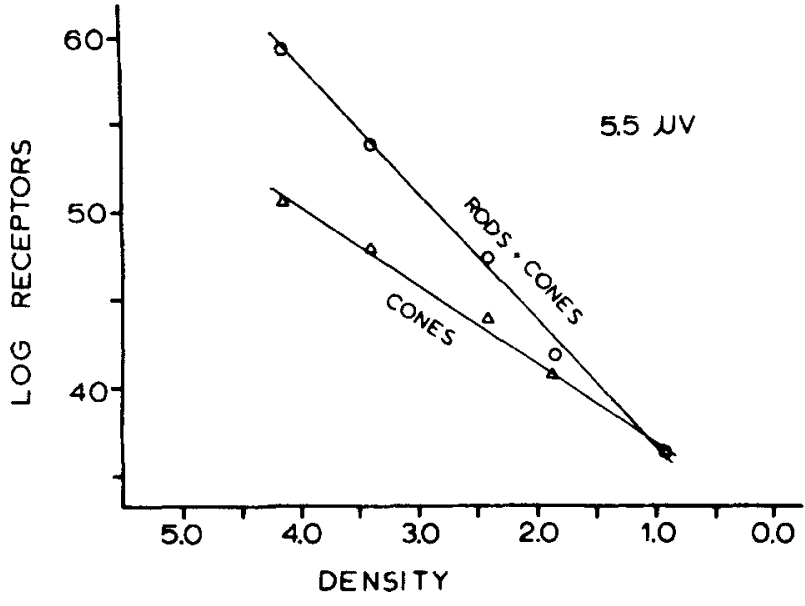

Figure 5. An estimate of the number of rod, and rod plus cone receptors in an area, plotted as a function of the luminance necessary to elicit a 5.5 -micro $V$ response.

much closer approximation to perfect summation. Interestingly enough, the area-luminance curves shown in Figure 4 all have slopes of about -.71, almost identical to the slope of the rod and cone function. The similarity of the two slopes would indicate that area in square millimeters is completely interchangeable with total receptor population within an area. The above analysis was applied to all of the criterion response levels, and the results were essentially identical to those described. Not shown in the figure is an additional comparison made by plotting the ganglion cell population in the five areas against the luminance values at $5.5 \mu \mathrm{V}$. An estimate of the ganglion cell population was obtained from the histological counts made by Van Buren (1963). The function obtained in this manner also fits a linear model, and the slope of this function was -.67. Again, this is in close agreement with the slopes of the area-luminance curves.

That the slopes of the functions do not indicate perfect summation is not too surprising. Very little is known of the mechanisms which contribute to the VEBR, and it is probably true that excitation and inhibition interact at the receptor level, the relay level, and the final cortical level of activity. It is possible that stray light may also have a limited effect. Thus, a perfect one-to-one correlation between receptor population and cortical activity may be an unreasonable assumption. More studies are needed to determine if stray light has an effect on the VEBR similar to that found in the ERG literature. Also, follow-up experiments might examine the effects of different surrounds in terms of size, luminance, and wavelength manipulations.

The results of the present study indicate that the VEBR technique can be a useful tool in the investigation of sensory function. Additional research 
is needed to determine if the functional relationship between area and luminance described in this paper holds true over a wider range of areas and luminance levels.

\section{REFERENCES}

Armington, J. C., \& Thiede, F. C. Effects of stimulus area and intensity upon the light-adapted electroretinogram. Journal of Experimental Psychology, 1954, 47, 329-334.

Armington, J. C. The electroretinogram, the visual evoked potential, and the area-luminance relation. Vision Research, $1968,8,263-276$.

Armington, J. C., Tepas, D. I., Kropfl, W. J., \& Hengst W. H. Summation of retinal potentials. Journal of the Optical Society of America, 1961, 51, 877-886.

Boynton, R. M., \& Riggs, L. A. The effect of stimulus area and intensity upon the human retinal response. Journal of Experimental Psychology, 1951, 42, 217-226.

DeVoe, R. G., Ripps, H., \& Vaughan, H. G. Cortical responses to stimulation of the human fovea. Vision Research, 1968, 8, 135-147.

Graham, C. H., \& Bartlett, N. B. The relation of size of stimulus and intensity in the human eye. II: Intensity thresholds for red and violet light. Journal of Experimental Psychology, $1939,24,574-586$.

Graham, C. H., Brown, R. H., \& Mote, F. A. The relation of sive of stimulus and intensity in the human eye. I: Intensity thresholds of white light. Journal of Experimental Psychology, $1939,24,555-573$.

Hillman, B. M. Relationship between stimulus size and threshold intensity in the fovea measured at four exposure times. Journal of the Optical Society of America, 1958, 48, 422-428.

JASPER, H. H. The ten-twenty electrode system of the inter- national federation. Electroencephalography \& Clinical Neurophysiology, 1958, 10, 371-375.

$\emptyset_{\text {sterberg, }}$. Topography of the layer of rods and cones in the human retina. Acta Ophthalmalogica, 1935, Supplement 6, 1-102.

PAGE, $H$. E. The relation between area of stimulation and intensity of light at various levels of visual excitation as measured by pupil constriction. Journal of Experimental Psychology, 1941, 29, 177-200.

TePas, D. I. Computer analysis of the electroencephalogram: Evoking, promoting, and provoking. Behavior Research Methods \& Instrumentation. 1974, 6, 95-110.

Tepas, D. I., \& Armington, J. C. Properties of evoked visual potentials. Vision Research, 1962, 2, 449-461.

Tepas, D. I., Boxerman, L. A., \& AnCH, A. M. Auditory evoked brain responses: Intensity functions from bipolar human scalp recordings. Perception \& Psychophysics, 1972, 11, 217-221.

Tepas, D. I., Guiteras, V. L., \& Klingaman, R. L. Variability of the human average evoked brain response to visual stimulation: A warning. Electroencephalography \& Clinical Neurophysiology, 1974, 36, 533-537.

Van Buren, J. M. The retinal ganglion cell layer. Springfield, Ill: Thomas, 1963.

Vaughan, H. G., JR. The perceptual and physiological significance of visual evoked responses recorded from the scalp in man. In H. M. Burian and J. H. Jacobson (Eds.), Clinical electroretinography (Supplement to Vision Research). New York: Pergamon, 1966

Walters, J. W. Scotopic vision at photopic levels? Vision Research, 1971, 11, 787.798.

(Received for publication April 4, 1974; revision accepted July 31,1974 .) 